\title{
Compare the efficiency of colposcopic VIA, VILI, LIQUIPREP TM and conventional Pap smear; as screening procedure for carcinoma cervix
}

\author{
Nithya Subbaiyan ${ }^{1}$, Sabari Kasinathan ${ }^{2 *}$
}

\begin{abstract}
${ }^{1}$ Department of Obstetrics and Gynecology, Government Villupuram Medical College and Hospital, Tamil Nadu, India
${ }^{2}$ K. R. K. Clinic, Gandhinagar, Virudhachalam, Tamil Nadu, India
\end{abstract}

Received: 10 December 2018

Accepted: 21 December 2018

\section{*Correspondence:}

Dr. Sabari Kasinathan,

E-mail: jabarali2009@gmail.com

Copyright: () the author(s), publisher and licensee Medip Academy. This is an open-access article distributed under the terms of the Creative Commons Attribution Non-Commercial License, which permits unrestricted non-commercial use, distribution, and reproduction in any medium, provided the original work is properly cited.

\begin{abstract}
Background: Cancer of the cervix is a leading cause of morbidity and mortality among women worldwide. Therefore, to curb the disease, there is a need to develop a screening test that has good sensitivity and specificity. The aim is to compare the efficiency of Colposcopic Via, Vili, Liquiprep TM and conventional pap smear; as screening procedure for carcinoma cervix.

Methods: This study was conducted in 100 women in the reproductive age. The pap smear and VIA are done in these cases. In positive cases, cervical biopsy and histopathological studies are done, the sensitivity and specificity of each test are determined and compared.

Results: In this study, more cases belonged to 30 - 45 years age group. Among 100 women, $15 \%$ cases had highgrade lesion. Among high-grade lesions group, one case that is $6.7 \%$ had CIN 1,73.3\% had CIN2/3 and 3cases i.e., $20 \%$ had cervicitis. Among 100 women, 1\% had ASCUS result, 10\% belongs to HSIL group, 39\% belongs to LSIL group and $2 \%$ had an unsatisfactory result.

Conclusions: The lack of an effective and implementable screening programme lead to reporting of advanced cases of $\mathrm{Ca}$ Cervix. If detected at CIN or early $\mathrm{Ca}$ cervix stage, effective treatment can be provided with encouraging results. Therefore, effective and implementable $\mathrm{Ca}$ Cervix screening need to be provided in our country.
\end{abstract}

Keywords: Ca cervix, Pap Smear, VIA

\section{INTRODUCTION}

The female genital tract is one of the most common places for primary malignant disease, among that cervix is the most common site affected by cancer. Worldwide cervical carcinoma is continuous to be a significant health care problem. ${ }^{1}$ In developing countries, cancer cervix is the most prevalent cancer. It has long preinvasive course and easily preventable by early detection and treatment. The gynaecologists and primary health care providers to women are familiar with vaccine programmes, screening techniques, diagnostic procedures, risk factors for cervical cancer and management of the pre-invasive disease. ${ }^{2,3}$ Improved patient education also contributed to improving the screening programmers. Preinvasive lesions progress slowly over 15-20 years to invasive cancer. Cervix is easily accessible to effective screening tests. It is also preventable malignancy, and screening tests identify the pre-invasive neoplastic condition in asymptomatic women. ${ }^{4}$ Colposcopy is a study of the subtle changes in the vascular pattern of the cervix, while cytology is a study of morphological changes in the cell. Excellent visualization of the cervix and vaginal portion is essential in colposcopy. The transformation zone should be circumferentially visualized. The use of a green filter helps to enhance the visualization of vascular 
abnormalities. After the application of 5\% acetic acid and Lugol's iodine over the cervix and the abnormal areas are identified and treated according to the findings. The colposcopy, Pap smear, Liquid-based cytology, colposcopy-directed biopsy are the four methods by which the cervix can be studied for evidence of early malignant change. They are simple OPD procedures requires no anesthesia. In present study, we compare the efficiency of colposcopic VIA, VILI, LIQUIPREP TM, and conventional pap smear; as a screening procedure for carcinoma cervix. We can also do the correlation between cytological and histological findings with cervical biopsy as the gold standard, the correlation between colposcopy and histological findings with cervical biopsy as the gold standard and compare the efficacy between conventional Pap smear with liquid-based cytology smear, compare the cytological and visual inspection findings with colposcopic directed biopsies., 5 Also, we evaluate the sensitivity and specificity of colposcopy versus cytological study in early detection of lesions. To compare the efficiency of Colposcopic Via, Vili, Liquiprep TM and conventional pap smear; as screening procedure for carcinoma cervix.

\section{METHODS}

A study was conducted in the Dept. of Obstetrics and Gynecology at Institute of Social Obstetrics and Government Kasturba Gandhi Hospital, Chennai during the year 2011-2012.

This study was conducted in 100 women in the reproductive age. A cervical smear was taken with cytobrush placed in os and rotate it 5 times over squamocolumnar junction and spread on a glass slide then fixed immediately in 95\% Alcohol, then decap the brush and put it into the thin prep preservative solution and sent for cytological analysis.

Colposcopic examination of unstained cervix and Inspection with the green filter was done; the Vascular pattern of cervix appeared in the green background when visualized through the green filter. Inspection of the cervix after $5 \%$ acetic acid application was done. After application, changes the color of the cervix after 10-30 seconds. The whole transformation zone must be visualized Surface contour, the margin of lesion and appearance of blood vessels are noted. Cervix was painted with Lugol's iodine in a 50\% dilution. Mahogany Brown staining occurs in the glycogen-rich squamous epithelium. The acetowhite area which was stained brown should indicate mature metaplastic epithelium. Mustard yellow or Saffron yellow indicates abnormal lesion. Interpretation of colposcopic findings was made by IFCPC nomenclature and SWEDE Scoring System.

\section{Exclusion criteria}

- Pregnant, history of Hysterectomy,

- History of cervical treatment for CIN,
- IUCD user, sexual Intercourse with spermicidal jelly,

- Douches/tampons 24 hours before pap smear examination.

\section{RESULTS}

Among 100 women, 7 were between 20-29 age group, 32 cases were between 30-39 years, and 12 were between 40 to 45 years. The incidence of CIN was $13.7 \%$ in $20-29$ years age group, $62.7 \%$ in $30-39$ years age group, $12 \%$ in $40-45$ years age group. $74 \%$ were married before 19 years, and $26 \%$ were married after 20 years. In this study incidence of CIN was $82.4 \%$ in less than 19 years age group and $17.6 \%$ in more than 20 years age group.

Table 1: Crosstabulation of colposcopy.

\begin{tabular}{|l|l|l|l|l|}
\hline Colposcopy & CIN & Cervicitis & Total & P value \\
\hline Positive & 48 & 10 & 58 & \\
\hline Negative & 3 & 39 & 42 & $<0.0001$ \\
\hline Total & 51 & 49 & 100 & \\
\hline
\end{tabular}

$1 \%$ belongs to below 5 years duration of the marriage, $14 \%$ belongs to $6-10$ years duration, $28 \%$ belongs to 11 15 years and $57 \%$ belongs to more than 15 years duration.

In this study CIN lesions, $9.8 \%$ belongs to 6-10 years duration and $29.4 \%$ belongs to $11-15$ years duration and $60.8 \%$ belongs to more than 15 years of duration. In this study $64.7 \%$ CIN lesions belong to class 5 socioeconomic group, $31.4 \%$ CIN lesions belongs to class 4 socio-economic group and $3.9 \%$ of CIN lesions belong to class 3 socio-economic group. 1\% was nullipara, $1 \%$ was primipara, $53 \%$ were belonging to para 2, 30\% were belonging to para 3 , and $15 \%$ were belonging to para 4 and above.

Table 2: Crosstabulation of Pap smear.

\begin{tabular}{|c|c|c|c|c|}
\hline \multirow{2}{*}{ Pap smear } & \multicolumn{2}{|c|}{ Biopsy } & \multirow{2}{*}{ Total } & \multirow{2}{*}{ P value } \\
\hline & CIN & Cervicitis & & \\
\hline Positive & 46 & 6 & 52 & \multirow{3}{*}{$<0.0001$} \\
\hline Negative & 5 & 43 & 48 & \\
\hline Total & 51 & 49 & 100 & \\
\hline
\end{tabular}

Among this CIN incidence was $51 \%$ in para $2,33.3 \%$ in para $3,15.7 \%$ in para 4 and above. Among para 3 in 30 cases incidence was $56.7 \%$. Among para 4 and above in 15 cases incidence was $53.3 \%$.

Table 3: Crosstabulation of liquid-based cytology.

\begin{tabular}{|l|l|l|l|l|l|}
\hline \multirow{2}{*}{$\begin{array}{l}\text { Liquid based } \\
\text { cytology }\end{array}$} & CIN & Cervicitis & Total & P value \\
\hline \multirow{2}{*}{ LBC } & Positive & 49 & 1 & 50 & \\
\cline { 2 - 5 } & Negative & 2 & 48 & 50 & $<0.0001$ \\
\hline Total & 51 & 49 & 100 & \\
\hline
\end{tabular}


Among 99 women, $21.2 \%$ were belonging to LCB 5 years group, $31.3 \%$ were belonging to LCB 6-10 years group, 36.4\% were belonging LCB 11-15 years group, and $11.1 \%$ were belonging to LCB more than 15 years group. Among that incidence of CIN was $21.6 \%$ in less than 5 years duration, $29.4 \%$ in $6-10$ years duration, $39.2 \%$ in $11-15$ years duration and $9.8 \%$ in more than 15 years duration group.

Table 4: Comparison of sensitivity and specificity of the tests.

\begin{tabular}{|l|l|l|l|}
\hline Test & Colposcopy & $\begin{array}{l}\text { Pap } \\
\text { smear }\end{array}$ & $\begin{array}{l}\text { Liquid based } \\
\text { cytology }\end{array}$ \\
\hline Sensitivity & 94.10 & 90.20 & 96.10 \\
\hline Specificity & 79.60 & 87.80 & 98 \\
\hline PPV & 82.80 & 88.50 & 98 \\
\hline NPV & 92.90 & 89.60 & 96 \\
\hline Accuracy & 87 & 89 & 97 \\
\hline
\end{tabular}

Among 100 women, in symptoms wise $63 \%$ had come with the symptom of Leucorrhoea, $25 \%$ had come with the symptom of lower abdomen pain, $7 \%$ had menstrual disturbances, $5 \%$ came for screening for cervical cancer. Among those CIN incidences was $60.8 \%$ in Leucorrhoea group, $25.5 \%$ in lower abdomen pain group, $9.8 \%$ in menstrual disturbances group, 3.90/0 in the screening group. Among 100 women, $61 \%$ of women had ectopy on per speculum examination findings and normal in $39 \%$ of patients. Among 100 women, CIN incidence was $62.7 \%$ in ectopy group and $37.3 \%$ in the normal group. Among 100 women, $15 \%$ of cases had a high-grade lesion, $43 \%$ case had a low-grade lesion, and $42 \%$ had the normal study. Among the high-grade lesions group, one case that is $6.7 \%$ had CIN $1,73.3 \%$ had CIN2/3 and 3 cases, i.e., $20 \%$ had cervicitis. Among low-grade lesions group, $83.7 \%$ had CINI, and $16.3 \%$ had cervicitis. Among the normal study group, $7.1 \%$ had CINI, and 92.9\% had cervicitis. Among 100 women had ASCUS, $8 \%$ had HSIL, had LSIL, $44 \%$ had NIL, and $4 \%$ had unsatisfactory reports. Among the ASCUS group, $40 \%$ had CINI lesion, $10 \%$ had a CIN2/3 lesion, and $50 \%$ had cervicitis.

Among the HSIL group, $12.5 \%$ had a lesion, and $87.5 \%$ had a CIN2/3 lesion. None had cervicitis. Among the LSIL group, 91.2\% had CINI lesion, 5.9\% had a CIN2/3 lesion, and $2.9 \%$ had cervicitis.

Among the NIL group, $2.3 \%$ had CINI lesion, none had a CIN2/3 lesion, and $97.7 \%$ had cervicitis. Among US group, $75 \%$ had CINI lesion, $25 \%$ had a CIN2/3 lesion. None had cervicitis. Among 100 women, 1\% had ASCUS result, $10 \%$ belongs to HSIL group, $39 \%$ belongs to LSIL group, and $2 \%$ had an unsatisfactory result. Among ASCUS group 100\% had CIN I lesion. Among HSIL group, had CIN 1 lesion and had CIN 11/111 lesions. Among the LSIL group, had CIN 1 lesions, $18.2 \%$ had CIN 11/111 lesions, and $2 \%$ had cervicitis.
Among NIL group $90 \%$ had cervicitis. Among the unsatisfactory group, 100\% had CIN 1 lesions.

\section{DISCUSSION}

Cervical cancer is the second most common cancer in developing countries. It is a preventable condition because it is associated with long precancerous duration making it amenable to screening and treatment. In this Study, screening was done in 100 women in reproductive age groups with the complaints of lower abdominal pain, white discharge, menstrual irregularities. Most of the women have ectopy of the cervix, and some have normal appearing cervix.

Regarding age distribution, high incidence of CIN was found among 30-45 years of the age group that is $86.2 \%$ of CIN lesions of all age groups. According to Kushtagi and Fernands study, the prevalence of CIN was higher in women over 30 years. $^{7}$ In Olaniyan's study, CIN was more prevalent in the age group of more than 35 years. ${ }^{8}$

Duration of marriage and sexual exposure had a distinct role in the genesis of CIN. In this study, the incidence of CIN was $60.8 \%$ among women were married for more than 15 years. Kushtagi et al. had demonstrated the severity of underlying CIN increased with the duration of the marriage and sexual exposure. ${ }^{7}$

Increase incidence of $\mathrm{CIN}$ is found in multiparous women, in this study parity 3 and above have more incidence of CIN lesions, i.e., 56.7\% in para 3 group, $53.3 \%$ in para 4 or more group. Shalini et al did a similar study, showed that the mean parity was 4.2 in patients with invasive cancer. ${ }^{9}$ Kushtagi and Fernands showed that the prevalence was higher in parity of more than two and Olaniyan's study also showed more cases of CIN were found with parity more than $4 .^{7,8}$

Socio-economic status plays an important role in the development of CIN lesions. In this study, increased incidence of CIN was found in class 5 socioeconomic status group. i.e., 64.7\%. Olaniyan's study also showed the definitive role of socio-economic status in the development of dysplasia, i.e., $80 \%$ of CIN I and $50 \%$ of CIN Il were from a lower income group. ${ }^{8}$ Poor hygiene, poor standard of living, multiple sexual partners and early exposure to sexual life are the factors associated with both lower income groups and cervical dysplasias.

In this study, $60.8 \%$ of women with leucorrhoea had CIN lesions. Leucorrhoea plays an important role in the development of CIN. Olaniyan et al also proved the above relation with leucorrhoea by $24 \%$ of vaginal discharge. ${ }^{8}$

Regarding the cervical appearances by clinical examination, the most common finding is cervical ectopy, where the columnar epithelium replaces the 
squamous epithelium. Ectopy was seen $61 \%$ women, among them $52.5 \%$ had lesions.

Among 100 women who underwent Colposcopy, 15\% cases had high-grade lesions, $43 \%$ cases had low-grade lesions, and $42 \%$ had a normal study. In high-grade lesion group, only $73.3 \%$ had CIN $2 / 3$ findings. In low grade' lesion group, only $83.7 \%$ had CIN I lesion. In the normal study group, $92.9 \%$ had cervicitis.

Table 5: Sensitivity and specificity of colposcopy by various authors.

\begin{tabular}{|l|l|l|}
\hline Author & Sensitivity (\%) & Specificity (\%) \\
\hline Pimple SA et al $^{10}$ & $58-74.7$ & $57.5-92.9$ \\
\hline $\begin{array}{l}\text { Olaniyan B } \\
\text { (Meta-analysis) }^{8}\end{array}$ & $87-99$ & $26-87$ \\
\hline Divya H et al & & \\
\hline Present Study & 70.8 & 95 \\
\hline
\end{tabular}

In this study correct estimation of Colposcopy was $87 \%$, the sensitivity was $94.1 \%$, and the specificity was $79.6 \%$. The positive predictive value of this test was $82.8 \%$, and the negative predictive value of this test was $92.9 \%$. (Table 1). Divya $\mathrm{H}$ et al, the study showed that the sensitivity was $70.8 \%$, the specificity was $95 \%$, PPV was $6219 \%$, 'NPV was $96.5 \% .^{11}$

Table 6: Comparison of study results with other studies.

\begin{tabular}{|l|l|l|}
\hline Author & Sensitivity (\%) & Specificity (\%) \\
\hline Donald A et al $^{12}$ & $87-88.6$ & $56.4-84.7$ \\
\hline Pimple SA et al $^{10}$ & 57.4 & 99.4 \\
\hline Divya H et al & & \\
\hline Present study & 83 & 98 \\
\hline
\end{tabular}

The meta-analysis study by Olaniyan B et al. showed that there was a high correlation between the Colposcopy and biopsy, the accuracy was $89 \%$. The present study also had an accuracy rate of $87 \% .^{8}$ The Colposcopy is the best screening tool because it had a low false negative rate, i.e., $3 \%$ and high accuracy rate of $87 \%$ which is well correlated with biopsy reports. In Pap smear study, among 10 women, $10 \%$ had ASCUS, $8 \%$ had HSIL, 34\% had LSIL, $44 \%$ had NIL, and $4 \%$ had unsatisfactory reports. In ASCUS group, had CIN 1 lesion, had CIN 2 lesions and $50 \%$ had cervicitis. In HSIL group, $87.5 \%$ had CIN 2/3 lesions. In LSIL group, 91.2\% had CIN 1 lesion. In NIL group, $97.7 \%$ had cervicitis.

In the present study, Pap smear had a sensitivity rate of $90.2 \%$. The specificity was $87.8 \%$, and the test of accuracy was $89 \%$. (Table 2) The specificity and sensitivity of the present study was correlated with Divya Hegde et al., study. ${ }^{11}$ The Pap smear had high sensitivity, specificity and accuracy rate. The reports are well correlated with biopsy reports. So, the Pap smear also the best screening tool. 'In LBC study, among 100 women, $1 \%$ had ASCUS, $10 \%$ had HSIL, had LSIL, had NIL, and
2\% had unsatisfactory reports. In ASCUS group, that one case showed CIN I result. In the HSIL group, had CIN 2/3 lesions. In LSIL group, $92.3 \%$ had CIN 1 lesion. In NIL group all cases had cervicitis.

The present study revealed a good correlation between LBC and Biopsy reports. It had a high sensitivity rate of $96.1 \%$ and specificity of $98 \%$. The PPV was $98 \%$, NPV was $96 \%$, and the accuracy rate was $97 \%$ (Table 3 ).

In this study, LBC is having higher sensitivity and specificity and accuracy rate than colposcopy and Pap smear. The sensitivity and specificity of combined Colposcopy and pap smear are almost equal to LBC results. Since $\mathrm{LBC}$ is more expensive, in our settings, we can use the combined Colposcopy and Pap smear for the cervical cancer screening (Table 4).

\section{CONCLUSION}

Early diagnosis and treatment is the best tool for the prevention of cancer cervix. Cancer cervix has long precancerous course, and it makes amenable to screening and treatment. This study concluded that accuracy, sensitivity, and specificity of LBC were higher than the Pap smear and Colposcopy, but it is more expensive. It is not used a screening procedure routinely in low resource settings, i.e., in developing and underdeveloped countries. Both Colposcopy and Pap smear combined results showed the best sensitivity and specificity. They are complementary to one another, so both of them can be used as the best screening tools.

Funding: No funding sources Conflict of interest: None declared

Ethical approval: The study was approved by the Institutional Ethics Committee

\section{REFERENCES}

1. Adadevoh SW, Forkoh BK, Cervical cancer Screening. Int J Gynaecol Obstet.1993;43(1):63-4

2. Basu P.S, Sankaranarayanan. R, Mandal. R, Roy. C, Das. P, Visual Inspection of Cervix with acetic add and cytology in the early detection of cervical neoplasia in Kolkotta - India. Int $\mathbf{J}$ Cancer. 2005;13(6):626-32.

3. Benedict JL, Matistic JP, Bertrand MA, An analysis of 84244 patients from theBritish Columbia Cytology-colposcopy program. J Gynecol 2004; 92(1):127-34.

4. McGraw SL, Ferrante JM. Update on prevention and screening of cervical cancer. World J Clin Oncol. 2014;5(4):744-52.

5. Juneja A, Murthy NS, Sharma S, Shukla DK, Roy M, Das DK. Selective cervical cytology screening: Discriminant analysis approach. Neoplasia. 1993;40(6):401-4. 
6. Sherwani RK, Khan T. Conventional pap smear and liquid based cytology for cervical cancer screeing. J Cytol. 2007;2(4):167-72.

7. Kushtagi P, Fernandez P, Significance of Persistent Inflammatory, Cervical smears in Sexually active women of reproductive age. J Obstet Gynecol lndia. 2002;52(1)124 - 6 .

8. Olaniyan OB. Validity of colposcopy in the diagnosis of early cervical neoplasia a review. African J Reproduct Health. 2002,6(3):59-69.

9. Shalini. R, Amita. S, Neera. M.A, How alarming is post coital bleeding - a cytologic, colposcopic and histological evaluation Gynaecol Obstet Invest. 1998;45(3):205 - 8.

10. Pimple SA, Amin G, Goswani S, Shastri SS. Evaluation of colposcopy vs cytology as secondary test to triage women found positive on visual inspection test. Indian J Cancer. 2010;47(2):308-13.
11. Divya H, Harish S, Prasanna KS, Supriya R. Diagnostic value of acetic acid comparing with conventional Pap smear in the detection of colposcopic biopsy-proved CIN. J Cancer Res Therapeut. 2011;7(4):454-8.

12. Donald A, Thomas T, Jaffery T, Ian S. Should liquid based cytology be performed prior to colposcopy? A comparison of the accuracy, unsatisfactory rates and cost in a referral setting. Aust NZ J Obstet Gynaecol. 2009;49(6):681-4.

Cite this article as: Subbaiyan N, Kasinathan S. Compare the efficiency of colposcopic VIA, VILI, LIQUIPREP TM and conventional Pap smear; as screening procedure for carcinoma cervix. Int $\mathbf{J}$ Reprod Contracept Obstet Gynecol 2019;8:69-73. 